\title{
Langmuir
}

pubs.acs.org/Langmuir

(C) 2010 American Chemical Society

\section{Large-Area Nanopatterning of Self-Assembled Monolayers of Alkanethiolates by Interferometric Lithography}

\author{
J. Adams,${ }^{\dagger}$ G. Tizazu, ${ }^{\dagger}$ Stefan Janusz,${ }^{\dagger}$ S. R. J. Brueck, ${ }^{\dagger}$ G. P. Lopez, ${ }^{*},, \perp$ and G. J. Leggett ${ }^{*}, \dagger$ \\ ${ }^{\dagger}$ Department of Chemistry, University of Sheffield, Brook Hill, Sheffield S3 7HF, U.K., "Center for High \\ Technology Materials, and ${ }^{\S}$ Center for Biomedical Engineering and Department of Chemical and \\ Nuclear Engineering, University of New Mexico, Albuquerque, New Mexico 87131, and \\ ${ }^{\perp}$ Department of Biomedical Engineering, Duke University, Durham, North Carolina 27708
}

Received May 11, 2010. Revised Manuscript Received June 25, 2010

\begin{abstract}
We demonstrate that interferometric lithography provides a fast, simple approach to the production of patterns in self-assembled monolayers (SAMs) with high resolution over square centimeter areas. As a proof of principle, two-beam interference patterns, formed using light from a frequency-doubled argon ion laser (244 nm), were used to pattern methyl-terminated SAMs on gold, facilitating the introduction of hydroxyl-terminated adsorbates and yielding patterns of surface free energy with a pitch of ca. $200 \mathrm{~nm}$. The photopatterning of SAMs on Pd has been demonstrated for the first time, with interferometric exposure yielding patterns of surface free energy with similar features sizes to those obtained on gold. Gold nanostructures were formed by exposing SAMs to UV interference patterns and then immersing the samples in an ethanolic solution of mercaptoethylamine, which etched the metal substrate in exposed areas while unoxidized thiols acted as a resist and protected the metal from dissolution. Macroscopically extended gold nanowires were fabricated using single exposures and arrays of $66 \mathrm{~nm}$ gold dots at $180 \mathrm{~nm}$ centers were formed using orthogonal exposures in a fast, simple process. Exposure of oligo(ethylene glycol)-terminated SAMs to UV light caused photodegradation of the protein-resistant tail groups in a substrate-independent process. In contrast to many protein patterning methods, which utilize multiple steps to control surface binding, this single step process introduced aldehyde functional groups to the SAM surface at exposures as low as $0.3 \mathrm{~J} \mathrm{~cm}^{-2}$, significantly less than the exposure required for oxidation of the thiol headgroup. Although interferometric methods rely upon a continuous gradient of exposure, it was possible to fabricate well-defined protein nanostructures by the introduction of aldheyde groups and removal of protein resistance in nanoscopic regions. Macroscopically extended, nanostructured assemblies of streptavidin were formed. Retention of functionality in the patterned materials was demonstrated by binding of biotinylated proteins.
\end{abstract}

\section{Introduction}

The development of methods for the organization of molecular structure at high spatial resolution remains a major goal in nanoscience. Self-assembled monolayers (SAMs) provide exquisite control of surface free energy, ${ }^{1}$ reactivity, biological interactions, ${ }^{2-7}$ and other properties, and they have thus attracted enormous interest for a wide variety of applications. A great deal of effort has been directed toward the patterning of SAMs. At the micrometer scale, microcontact printing $(\mu \mathrm{CP})^{8,9}$ remains the most widely used technique, providing rapid reproduction of patterns with high fidelity. Although less widely used, photopatterning ${ }^{10-12}$ also provides a

*Corresponding authors. E-mail: graham.leggett@sheffield.ac.uk (G.J.L.); gabriel.lopez@duke.edu (G.P.L.).

(1) Bain, C. D.; Troughton, E. B.; Tao, Y.-T.; Evall, J.; Whitesides, G. M.; Nuzzo, R. G. J. Am. Chem. Soc. 1989, 111, 321.

(2) Lopez, G. P.; Albers, M. W.; Schreiber, S. L.; Carroll, R.; Peralta, E.; Whitesides, G. M. J. Am. Chem. Soc. 1993, 115, 5877.

(3) Lopez, G. P.; Biebuyck, H. A.; Haerter, R.; Kumar, A.; Whitesides, G. M. J. Am. Chem. Soc. 1993, 115, 10774.

(4) Prime, K. L.; Whitesides, G. M. Science 1991, 252, 1164.

(5) Ostuni, E.; Chapman, R. G.; Holmlin, E. R.; Takayama, S.; Whitesides, G. M. Langmuir 2001, 17, 5605,

(6) Chen, C. S.; Mrksich, M.; Huang, S.; Whitesides, G. M.; Ingber, D. E. Science 1997, 276, 1425.

(7) Singhvi, R.; Kumar, A.; Lopez, G. P.; Stephanopoulos, G. N.; Wang, D. I. C.; Whitesides, G. M.; Ingber, D. E. Science 1994, 264, 696.

(8) Xia, Y.; Whitesides, G. M. Angew. Chem., Int. Ed. 1998, 37, 550.

(9) Love, J. C.; Estroff, L. A.; Kriebel, J. K.; Nuzzo, R. G.; Whitesides, G. M. Chem. Rev. 2005, 105, 1103.

(10) Tarlov, M. J.; Burgess, D. R. F.; Gillen, G. J. Am. Chem. Soc. 1993, 115, 5305 .

(11) Huang, J.; Hemminger, J. C. J. Am. Chem. Soc. 1993, 115, 2243.

(12) Leggett, G. J. Chem. Soc. Rev. 2006, 35, 1150. simple and rapid route to the fabrication of micropatterned SAMs. At the nanometer scale, there has been a great deal of interest in the utilization of scanning probe-based methods, including dippen nanolithography, ${ }^{13-16}$ nanoshaving/nanografting, ${ }^{17-20}$ and near-field optical techniques. ${ }^{21-25}$ Such approaches yield extremely high resolution (for example, $9 \mathrm{~nm}$ by scanning near-field photolithography ${ }^{26}$ ), but local probe techniques provide a slow means of fabricating structures larger than a single scan area. Parallelization of scanning probe methods ${ }^{27}$ offers one solution to

(13) Piner, R. D.; Zhu, J.; Xu, F.; Hong, S.; Mirkin, C. A. Science 1999, 283, 661. (14) Lee, K.-B.; Park, S.-J.; Mirkin, C. A.; Smith, J. C.; Mrksich, M. Science 2002, 295, 1702 .

(15) Lee, K.-B.; Lim, J.-H.; Mirkin, C. A. J. Am. Chem. Soc. 2003, 125, 5588

(16) Hyun, J.; Ahn, S. J.; Lee, W. K.; Chilkoti, A.; Zauscher, S. Nano Lett. 2002, 2,1203 .

(17) Wadu-Mesthrige, K.; Amro, N. A.; Garno, J. C.; Xu, S.; Liu, G.-Y. Biophys. J. 2001, 80, 1891.

(18) Amro, N. A.; Xu, S.; Liu, G.-Y. Langmuir 2000, 16, 3006

(19) Zhou, D. S., K.; Abell, C.; Rayment, T. Angew. Chem., Int. Ed. 2003, 42, 4934 .

(20) Zhou, D.; Wang, X.; Birch, L.; Rayment, T.; Abell, C. Langmuir 2003, 19, 10557.

(21) Sun, S.; Chong, K. S. L.; Leggett, G. J. J. Am. Chem. Soc. 2002, 124, 2414

(22) Sun, S.; Leggett, G. J. Nano Lett. 2002, 2, 1223.

(23) Sun, S.; Leggett, G. J. Nano Lett. 2004, 4, 1381.

(24) Reynolds, N. P.; Janusz, S. J.; Escalante-Marun, M.; Timney, J.; Ducker, R. E.; Olsen, J. D.; Otto, C.; Subramanian, V.; Leggett, G. J.; Hunter, C. N. J. Am. Chem. Soc. 2007, 129, 14625.

(25) Reynolds, N. P.; Tucker, J. D.; Davison, P. A.; Timney, J. A.; Hunter, C. N.; Leggett, G. J. J. Am. Chem. Soc. 2009, 131, 896.

(26) Montague, M.; Ducker, R. E.; Chong, K. S. L.; Manning, R. J.; Rutten, F. J. M.; Davies, M. C.; Leggett, G. J. Langmuir 2007, 23, 7328.

(27) Vettiger, P.; Despont, M.; Drechsler, U.; Durig, U.; Haberle, W.; Lutwyche,

M. I.; Rothuizen, H.; Stuts, R.; Widmer, R.; Binnig, G. K. IBM J. Res. Dev. 2000, 44,323 . 
this difficulty but nevertheless requires specialized apparatus. Electron-beam lithography is the most well-established nanofabrication tool and has been used to pattern SAMs at extremely high resolution, ${ }^{28-31}$ but in addition to the high cost and complexity of the necessary infrastructure, electron beam techniques do not currently provide a convenient means of fabricating large numbers of patterned samples, potentially necessary for many putative applications of patterned SAMs in nanotechnology (for example, arrays of biofunctional sensors).

Here we describe a simple alternate approach: interferometric exposure. ${ }^{32}$ Other than a UV laser, it requires only very modest resources, and it should be capable of exploitation by many laboratories not otherwise equipped with infrastructure for nanofabrication. Previously, extreme-UV radiation from a synchrotron light source has been used to modify monolayers with photosensitive nirobenzyl terminal groups. ${ }^{33}$ However, in the present study, a very simple benchtop apparatus is used. An important benefit of interferometric methods is that they facilitate patterning over large (square centimeter) areas, thus making them ideal for adaptation to biological applications (for example, control of cell-surface interactions). Our work builds on two earlier observations. First, photochemical modification of SAMs provides a variety of flexible approaches to surface patterning. For example, alkanethiolates on gold are photo-oxidized ${ }^{34}$ to yield alkylsulfonate species ${ }^{10,11,35,36}$ that may be displaced readily by a second, solution-phase thiol, yielding a chemical pattern or, by a solutionphase etchant, to create three-dimensional structures. Second, using near-field optical techniques, the same simple methodologies may be used for nanofabrication by exposing the SAM to the evanescent field associated with the tip of a scanning near-field optical microscope (SNOM). ${ }^{12,21,23}$ Such approaches yield a resolution that is, routinely, of the order of a few tens of nanometers and, at best, as good as $9 \mathrm{~nm}^{26}$ However, SNOM instrumentation is not readily available, and SNOM suffers the limitation of being a local probe technique. The keys to achieving such high spatial resolution during near-field patterning of SAMs are, first, the effective confinement of the excitation and, second, the fact that the resist is a monolayer; i.e., the thickness of the dielectric layer is minimized. In the present work, we have replaced the SNOM with a two-beam interference system. ${ }^{32,37}$ The interfering laser beams yield a pattern of intensity that has a period $\lambda / 2 n \sin \theta$. A single exposure yields parallel lines, while orthogonal exposures define a grid of exposure. ${ }^{32}$ By controlling the laser beam intensity, duration of exposure, and the angle between the interfering beams, it is possible to exercise fine control over the pattern of modification that results. Here we show that for a SAM resist it is possible to yield high spatial resolution.

A specific goal in the present study was to develop a methodology that facilitated control of protein organization at high spatial resolution. The biggest challenge in protein patterning is the control of nonspecific adsorption. Here we utilize SAMs formed from oligo(ethylene glycol)-terminated alkanethiolates, which have

(28) Zharnikov, M.; Grunze, M. J. Vac. Sci. Technol. B 2002, 20, 1793.

(29) Krakert, S.; Ballav, N.; Zharnikov, M.; Terfort, A. Phys. Chem. Chem Phys. 2010, 12, 507.

(30) Ballav, N.; Thomas, H.; Winkler, T.; Terfort, A.; Zharnikov, M. Angew. Chem., Int. Ed. 2009, 48, 5833.

(31) Ballav, N.; Terfort, A.; Zharnikov, M. Langmuir 2009, 25, 9189.

(32) Brueck, S. R. J. Proc. IEEE 2005, 93, 1704.

(33) Turchanin, A.; Schnietz, M.; El-Desawy, M.; Solak, H. H.; David, C.; Golzhauser, A. Small 2007, 3, 2114.

(34) Brewer, N. J.; Rawsterne, R. E.; Kothari, S.; Leggett, G. J. J. Am. Chem. Soc. 2001, 123, 4089 .

(35) Hutt, D. A.; Leggett, G. J. J. Phys. Chem. 1996, 100, 6657.

(36) Hutt, D. A.; Cooper, E.; Leggett, G. J. J. Phys. Chem. B 1998, 102, 174.

(37) Lu, C.; Lipson, R. H. Laser Photon. Rev. 2009, 1. previously been shown to be highly resistant to protein adsorption. ${ }^{3,5}$ It has recently been shown that such monolayers may be photodegraded by exposure to UV light, ${ }^{38}$ removing their protein resistance and facilitating the binding of functional molecules. Here we show that such approaches are compatible with interferometric lithography, yielding, in a single step, photoconversion of protein-resistant regions to protein binding ones with high spatial resolution over large areas.

\section{Experimental Section}

Preparation of SAMs. Substrates were prepared by evaporation of ca. $25 \mathrm{~nm}$ of Au or Pd onto Cr-primed glass slides (Chance Proper, No. 1, Agar, Cambridge, UK). Substrates were immersed in $1 \mathrm{mM}$ solutions of the appropriate thiol in degassed ethanol. Octadecanethiol, mercaptoundecanol, mercaptoethylamine, mercaptoundec-11-yl)tri(ethylene glycol) $\left(\mathrm{C}_{11}(\mathrm{OEG})_{3}\right)$, and HPLC purity ethanol were purchased from Sigma Chemical Co. All glassware was cleaned using piranha solution, a 3:7 mixture of $30 \%$ hydrogen peroxide and concentrated sulfuric acid. Piranha solution is a very strong oxidizing agent and should be used with great caution.

SAM Photo-oxidation. The photo-oxidation of SAMs of alkanethiolates on Au has previously been studied extensively by spectroscopic methods. X-ray photoelectron spectroscopy was used to confirm that oxidation was accompanied by the characteristic changes in the $\mathrm{S} 2 \mathrm{p}$ region reported in earlier studies. ${ }^{31}$ SAMs of octadecanethiolate (henceforth $\mathrm{C}_{17} \mathrm{CH}_{3}$ ) on gold were exposed to light from a frequency-doubled argon ion laser (Coherent FreD 300C) using a simple two-beam apparatus, ${ }^{32}$ consisting of a sample stage and mirror separated by an angle $2 \theta$.

Surface Analysis. Friction force microscopy measurements were carried out on a Digital Instruments Nanoscope Multimode IIIa atomic force microscope (Digital Instruments, Cambridge, UK). The probes used were silicon nitride Nanoprobes (Digital Instruments, Cambridge, UK). The nominal force constants of these probes were 0.06 or $0.12 \mathrm{~N} \mathrm{~m}^{-1}$. XPS characterization was carried out using a Kratos Axis ULTRA “DLD” X-ray photoelectron spectrometer, equipped with a monochromatic Al K $\alpha$ $\mathrm{X}$-ray source $(h v=1486.6 \mathrm{eV})$ operating with a base pressure in the range $10^{-8}-10^{-10} \mathrm{mbar}$. All samples were run as insulators, unless stated otherwise. In order to minimize X-ray-induced damage, the $\mathrm{X}$-ray source was operated at a relatively low output power of $60 \mathrm{~W}$. Here high-resolution scans of the $\mathrm{C}$ 1s region were acquired at a pass energy of $20 \mathrm{eV}$ and a step size (resolution) of $0.1 \mathrm{eV}$. Survey scans were acquired with a pass energy of $160 \mathrm{eV}$ and a step size of $1.0 \mathrm{eV}$.

Etching. Photopatterned films were etched using a s $1 \mathrm{mM}$ solution of mercaptoethylamine in ethanol, to which one drop of dilute ammonium hydroxide had been added. The samples were immersed for ca. $2 \mathrm{~h}$ (or until the sample was transparent to the naked eye). After removal from the etch solution, the samples were rinsed in ethanol and dried prior to analysis.

Protein Adsorption. Bovine serum albumin, streptavidin, and mouse antihuman IgG were obtained from Sigma and prepared at a concentration of $1 \mu \mathrm{g} \mathrm{mL}^{-1}$ in phosphate-buffered saline solution. Samples were immersed in the solutions following lithographic exposure and subsequently rinsed with buffer and dried prior to AFM analysis.

\section{Results and Discussion}

SAM Photo-oxidation and Solution-Phase Replacement on Au and Pd. Figure 1 shows a schematic diagram of the Fresnel mirror apparatus used in this work. ${ }^{32}$ Half of the expanded laser beam fell directly onto the sample, and half fell onto the mirror,

(38) Ducker, R. E.; Janusz, S. J.; Sun, S.; Leggett, G. J. J. Am. Chem. Soc. 2007, 129,14842 


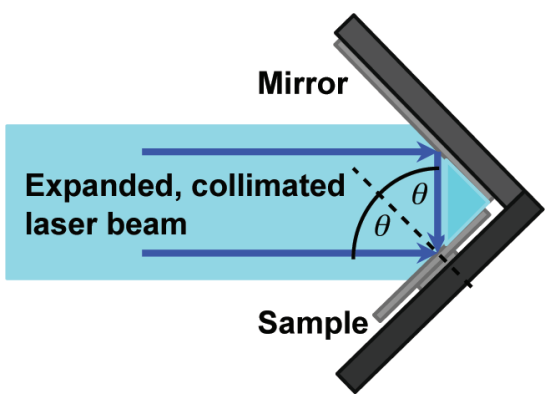

Figure 1. Schematic diagram showing the two-beam interference apparatus.

from where it was reflected onto the sample, interfering with the other half of the beam and yielding a sinusoidal pattern of exposure.

Photopatterning of SAMs on submicrometer length scales has previously been carried out by proximal exposure, either using a mask placed in contact with the sample surface or by exposure to a near-field probe. In contrast, in IL, a continuous gradient of intensity is utilized to expose the resist in projection mode; the effect of this mode of exposure on SAM chemistry was not known. The factors affecting line edge roughness were also not known. To examine these issues, SAMs of octadecanethiol, henceforth $\mathrm{C}_{17} \mathrm{CH}_{3}$, were photopatterned by exposure for $10 \mathrm{~min}$ in the interferometer, after which the sample was immersed in a solution of mercaptoundecanol (henceforth $\mathrm{C}_{11} \mathrm{OH}$ ) for $2 \mathrm{~h}$, leading to displacement of photo-oxidized adsorbates. Subsequently, the sample was rinsed in ethanol and imaged by friction force microscopy (FFM), a variant of atomic force microscopy (AFM) in which lateral deflections of the AFM probe are measured and used to track changes in the frictional properties of the surface. In previous studies it has been established that methyl- and hydroxyl-terminated regions of the sample yield differential contrast in FFM images ${ }^{39-41}$ because for a silicon nitride probe, there is relatively strong adhesion to polar surfaces, yielding a higher rate of energy dissipation and hence a larger friction force at a given load, while adhesion, and hence the friction force at the same load, are correspondingly smaller for nonpolar surfaces. ${ }^{41-43}$

Figure 2a shows an FFM image of a pattern created using IL. Bright, parallel, lines are observed that correspond to regions where the $\mathrm{C}_{17} \mathrm{CH}_{3}$ adsorbates have been photo-oxidized and replaced by $\mathrm{C}_{11} \mathrm{OH}$. The dark regions in between represent lines of $\mathrm{C}_{17} \mathrm{CH}_{3}$. The pitch in Figure $2 \mathrm{a}$ is $192 \mathrm{~nm}$. In contrast to a mask-based exposure method, IL yields a continuous, sinusoidal gradient of exposure. However, the exposure was adjusted so that maxima in the IL pattern yielded complete photo-oxidation, and the minima represented regions where modification was negligible. Analysis of line sections through the FFM image indicated that the full width at half-maximum (fwhm) was $90 \mathrm{~nm}$. A characteristic of the features in Figure 2a, besides the very good spatial resolution, is the exceptional uniformity of the structures over extended distances. Random sampling of an area $5 \times 5 \mathrm{~mm}^{2}$ yielded identical structures. Although scanning probe-based techniques offer slightly higher spatial resolution for SAM patterning, none offers the capacity to pattern such a large area, with such high density and regularity, so quickly.

SAMs may be formed by the adsorption of alkanethiols onto palladium. SAMs on Pd offer a variety of potential advantages

(39) Frisbie, C. D.; Rozsnyai, L. F.; Noy, A.; Wrighton, M. S.; Lieber, C. M. Science 1995, 265, 2071.

(40) McDermott, M. T.; Green, J.-B. D.; Porter, M. D. Langmuir 1997, 13, 2504

(41) Brewer, N. J.; Beake, B. D.; Leggett, G. J. Langmuir 2001, 16, 735.

(42) Chong, K. S. L.; Sun, S.; Leggett, G. J. Langmuir 2005, 21, 3903.

(43) Whittle, T. J.; Leggett, G. J. Langmuir 2009, 25, 9182.
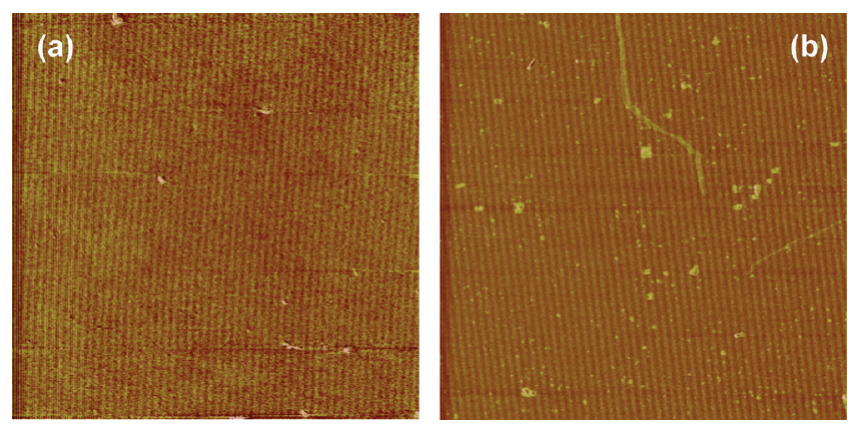

Figure 2. (a) $10 \times 10 \mu \mathrm{m}^{2} \mathrm{FFM}$ image of an IL pattern formed by exposure of $\mathrm{a}_{17} \mathrm{CH}_{3} \mathrm{SAM}$ on $\mathrm{Au}$ followed by immersion in a solution of $\mathrm{C}_{11} \mathrm{OH}$. $z$-contrast range: $0-200 \mathrm{mV}$ dark to bright. (b) $10 \times$ $10 \mu \mathrm{m}^{2}$ FFM image of an IL pattern formed by exposure of a $\mathrm{C}_{17} \mathrm{CH}_{3} \mathrm{SAM}$ on $\mathrm{Pd}$ followed by immersion in a solution of $\mathrm{C}_{11} \mathrm{OH}$. $z$-contrast range: $0-200 \mathrm{mV}$ dark to bright.

(for example, Pd is more catalytically active than gold, is compatible with CMOS technology, and, because of its small grain size, forms a very effective etch resist). ${ }^{9,44,45}$ Work by Love et al. suggests that SAMs on Pd are closer, structurally speaking, to SAMs on silver than gold, exhibiting a tilt angle of $19^{\circ}$ and probably adopting closer packing. ${ }^{45}$ Using X-ray photoelectron spectroscopy, we confirmed that SAMs of alkanethiols on Pd are photo-oxidized on exposure to light with a wavelength of $244 \mathrm{~nm}$. For the virgin material, an S 2p peak was observed at $162.9 \mathrm{eV}$. After exposure, a new peak was observed at $168.8 \mathrm{eV}$, attributed to the alkylsulfonate species. This peak increased in intensity with increasing exposure, and the thiolate sulfur species, at $162.9 \mathrm{eV}$, decreased in intensity. Figure $2 \mathrm{~b}$ shows a patterned sample fabricated using interferometric exposure of a $\mathrm{SAM}$ of $\mathrm{C}_{11} \mathrm{CH}_{3}$ on $\mathrm{Pd}$. Following exposure, the sample was immersed in a solution of $\mathrm{C}_{11} \mathrm{OH}$, resulting in displacement of the oxidation products by the polar thiol, just as for the sample in Figure 2a. Again, clear contrast is observed in the friction image between the regions exposed to the maxima and minima in the interference pattern at a pitch of $192 \mathrm{~nm}$.

Fabrication of Gold Nanostructures Using IL of SAMs and Solution-Phase Etching. There has been a great deal of interest in the use of metallic nanostructures for a range of applications, including the exploitation of plasmonic effects for biological analysis. ${ }^{46-50}$ Existing methods for the fabrication of gold nanostructures include colloidal lithography, electron beam lithography, and nanoimprinting, but each is subject to limitations. ${ }^{51}$ Colloidal lithography ${ }^{52}$ is simple and inexpensive but offers limited capacity for control of their periodicity, feature dimensions, and geometry, while electron beam lithography relies upon expensive infrastructure and nanoimprinting requires the prior fabrication of a mask using costly processes. At the micrometer scale, microcontact printing has been an enormously valuable tool because it provides a rapid, inexpensive means of patterning SAM resists

(44) Love, J. C.; Wolfe, D. B.; Chabinyc, M. L.; Paul, K. E.; Whitesides, G. M. J. Am. Chem. Soc. 2002, 124, 1576.

(45) Love, J. C.; Wolfe, D. B.; R. Haasch, R.; Chabinyc, M. L.; Paul, K. E.; Whitesides, G. M.; Nuzzo, R. G. J. Am. Chem. Soc. 2003, 125, 259

(46) Nie, S.; Emory, S. R. Science 1997, 275, 1102.

(47) Cao, Y. C.; Jin, R.; Mirkin, C. A. Science 2002, 297, 1536.

(48) Graham, D.; Thompson, D. G.; Smith, W. E.; Faulds, K. Nature Nanotechnol. 2008, 3, 548 .

(49) Anker, J. N.; Hall, W. P.; Lyandres, O.; Shah, N. C.; Zhao, J.; Van Duyne, R. P. Nature Mater. 2008, 7, 442.

(50) Jonsson, M. P.; Jonsson, P.; Dahlin, A. B.; Hook, F. Nano Lett. 2007, 7, 3462 .

(51) Nagpal, P.; Lindquist, N. C.; Oh, S.-H.; Norris, D. J. Science 2009, 325, 594

(52) Michel, R.; Reviakine, I.; Sutherland, D.; Fokas, C.; Csucs, G.; Danuser, G.; Spencer, N. D.; Textor, M. Langmuir 2002, 18, 8580 . 

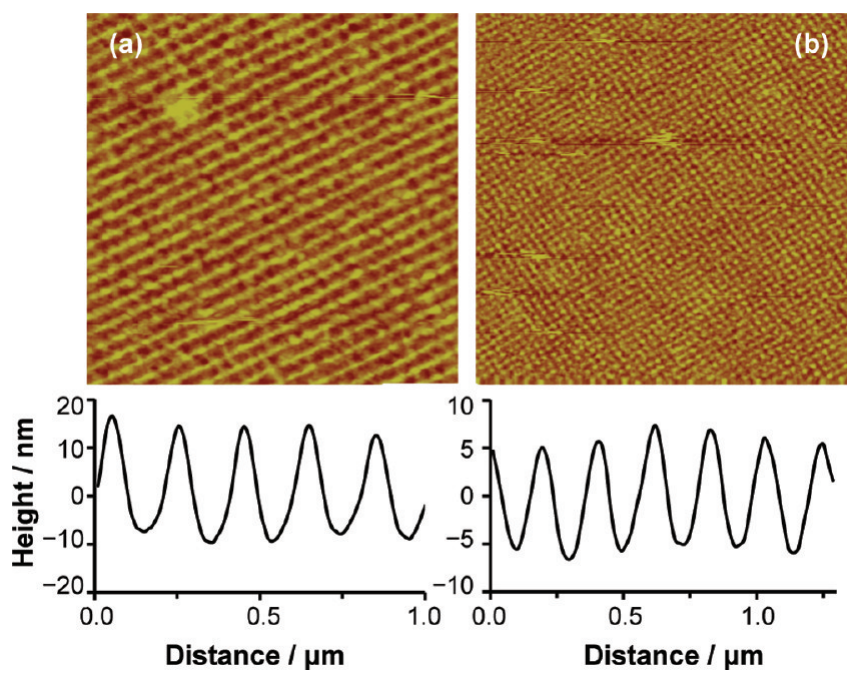

Figure 3. AFM topographical images of gold nanostructures formed by solution-phase etching of IL-patterned SAMs on Au and line sections through representative regions of the respective images. Images sizes: (a) $4.6 \times 4.6 \mu \mathrm{m}^{2}, z$-contrast range $0-50 \mathrm{~nm}$ dark to bright; (b) $10 \times 10 \mu \mathrm{m}^{2}, z$-contrast range $0-50 \mathrm{~nm}$ dark to bright.

that may be used for the solution-phase etching of gold, ${ }^{8,9}$ but its resolution is limited. The utility of IL for the rapid fabrication of gold nanostructures over macroscopic areas was thus tested by combining interferometric patterning of SAMs with solutionphase etching.

The fabrication of close-packed arrays of gold nanowires and nanocrystals is particularly challenging because the common etch agents are isotropic in action (hence creating the potential for undercutting) and yield comparatively poor line-edge roughness (especially for polycrystalline films with a substantial grain size). An organic etch solution, mercaptoethylamine in ethanol, was selected for the present study. There was one previous report of the use of this very mild reagent to etch trenches into gold films, following their patterning by near-field lithography, ${ }^{53}$ but its utility for etching close-packed structures in dense arrays was not known. Samples were exposed to UV light using the interferometer, and following exposure, they were etched by immersion in a $100 \mathrm{mM}$ solution of mercaptoethylamine in ethanol to which a trace amount of ammonia had been added.

Figure 3a shows a contact mode AFM image of parallel gold wires formed using IL. A single exposure was carried out of a hexadecanethiol SAM on gold supported on a glass microscope slide, and this was followed by immersion of the sample in the mercaptoethylamine/ethanol etch solution. In regions of the pattern where the adsorbates had been fully photo-oxidized, the etch solution displaced the sulfonate oxidation products from the surface and eroded the underlying gold. In regions protected by unoxidized thiols, the gold film was left intact. After etching the microscope slide was transparent, suggesting that the etch process removed the full thickness of the Au film from the exposed areas. Analysis of a line section through Figure $3 \mathrm{a}$ yielded a height difference of $23 \mathrm{~nm}$ between the maxima and minima, with a pitch of $180 \mathrm{~nm}$ and a fwhm of $72 \mathrm{~nm}$. This height difference corresponds to the thickness of the gold film used.

Arrays of gold dots were fabricated by carrying out two orthogonal exposures of a hexadecanethiol SAM on gold. The sample was again etched with mercaptoethylamine in ethanol.

(53) Ducker, R. E.; Leggett, G. J. J. Am. Chem. Soc. 2006, 128, 392.
Figure $3 \mathrm{~b}$ shows a contact mode AFM image of the resulting structure, which consisted of an array of gold "dots" with a mean fwhm of $66 \mathrm{~nm}$ at $180 \mathrm{~nm}$ centers.

In previous studies of the solution-phase etching of photopatterned SAMs, the use of short-chain thiols was found to lead to some removal of gold from regions protected by unoxidized adsorbates because they possess higher defect densities than monolayers formed from longer adsorbates such as hexadecanethiol. ${ }^{53}$ Despite the exposure of the sample to a continuous gradient of exposure in the present study, the regular periodic nature of the cross sections through the images in Figure 3 indicates that etching is largely confined to regions of extensive photo-oxidation (the maxima in the interference pattern). It thus does not seem that the low intensity of exposure in other regions creates adequately large numbers of defects to initiate etching. In addition, the mild organic etch appears not to have led to excessive line edge roughness. The structures shown in Figure 3 were formed by etching polycrystalline gold films, with a grain size that was comparatively large (i.e., not dissimilar to the fwhm of the resulting structures). Control of the grain size, or the use of an epitaxially deposited substrate, may yield significant further improvements in edge definition.

The data in Figure 3 indicate that IL exposure of SAMs, combined with wet etching, provides a means of fabricating nanometerscale gold structures that are suitable for plasmonic applications. In contrast to electron beam lithography, however, only modest infrastructure is needed. Moreover, the sizes and dimensions of the resulting nanostructures are readily controlled by varying $\theta$ (which controls the pitch and line width), the number of exposures, and the angle of rotation of the sample between exposures.

Protein Nanopatterning. There are two principal requirements for protein patterning. First, control of nonspecific adsorption is critical because proteins adsorb strongly and irreversibly to most surfaces. Extensive studies of oligo(ethylene glycol) (OEG)terminated SAMs have demonstrated that they exhibit exceptional resistance to protein adsorption. ${ }^{4,54}$ A number of protein patterning methods have been developed that rely upon the selective deposition or removal of OEG-terminated adsorbates. ${ }^{2,3,14}$ Second, a means is required to couple proteins to the remaining regions of the surface. Many of the methods used to immobilize proteins at surfaces involve multiple steps, creating further difficulties in processing, in addition to those associated with the definition of protein-resistant regions.

Recently, it was reported that monolayers of OEG-terminated SAMs may be degraded by exposure to UV light to yield aldehydefunctionalized surfaces that bind amines strongly via imine bond formation. ${ }^{38}$ This approach is very attractive because it provides a one-step conversion of a protein-resistant surface to a proteinbinding one. Moreover, in contrast to methods that rely upon the selective deposition or removal of thiols, it is also a substrateindependent process: in principle, it should be applicable to any OEG-functionalized surface. Here, the potential for using IL to selectively photodegrade OEG-terminated SAMs with nanometer resolution over macroscopic areas has been investigated.

To determine the correct exposure, a quantitative investigation of the development of surface chemical composition with UV exposure was carried out by XPS. Monolayers of (1-mercaptoundec-11-yl)tri(ethylene glycol) were exposed to UV light, and their $\mathrm{C}$ 1s spectra were recorded. Figure $4 \mathrm{a}$ shows the $\mathrm{C}$ 1s spectrum of a virgin sample. The spectrum has been fitted using two components: an aliphatic carbon component corresponding to the

54) Pale-Grosdemange, C.; Simon, E. S.; Prime, K. L.; Whitesides, G. M. J. Am. Chem. Soc. 1991, 113, 12. 

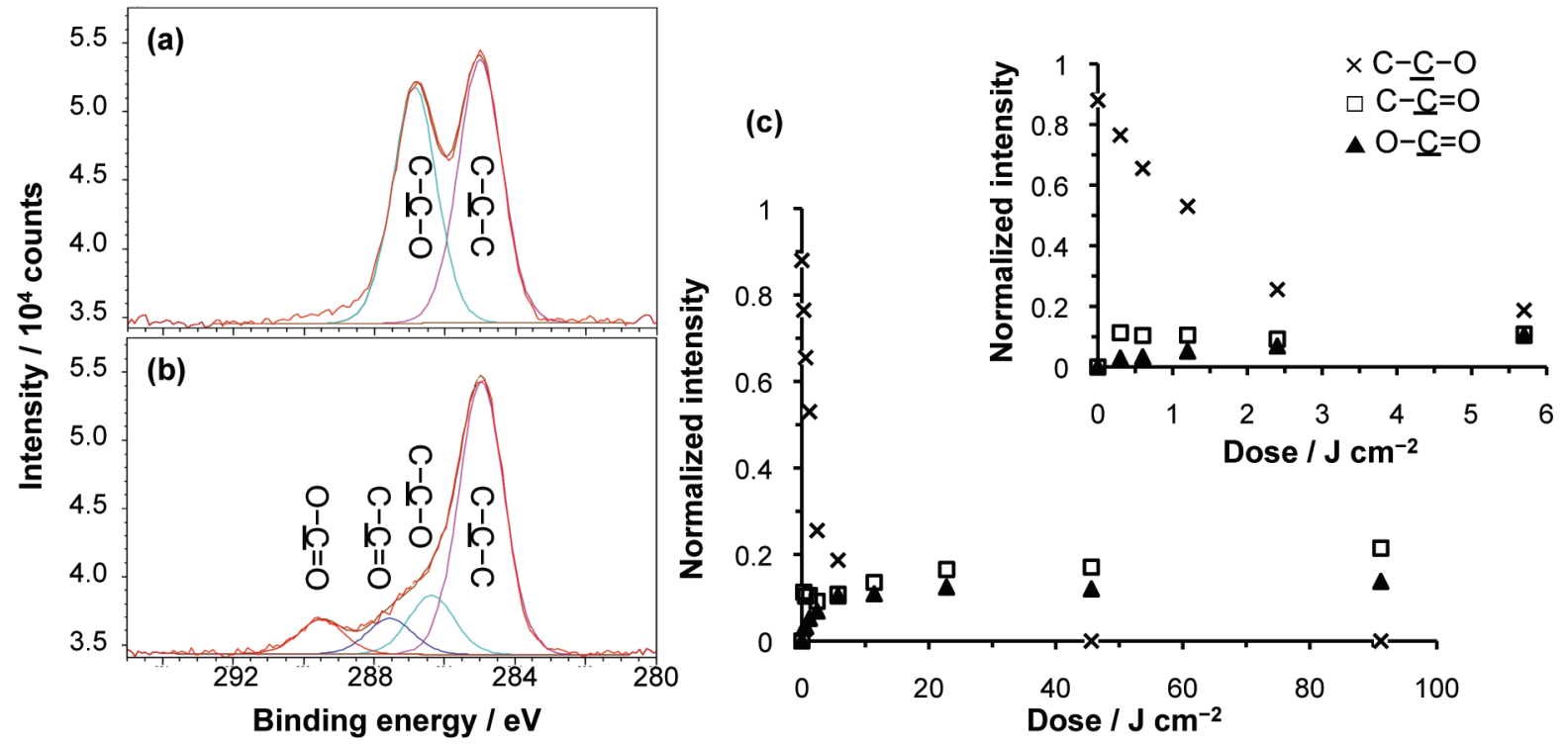

Figure 4. (a) XPS C 1s spectrum for a virgin OEG-terminated SAM. (b) C 1s spectrum for a sample exposed to a uniform UV dose of $2.2 \mathrm{~J} \mathrm{~cm}^{-2}$. (c) Variation with UV exposure of the areas (normalized to the area of the $\mathrm{C}-C-\mathrm{C}$ peak) of the main components in the $\mathrm{C} 1 \mathrm{~s}$ spectra of OEG-terminated SAMs. Error bars are estimated to be smaller than the symbols used.
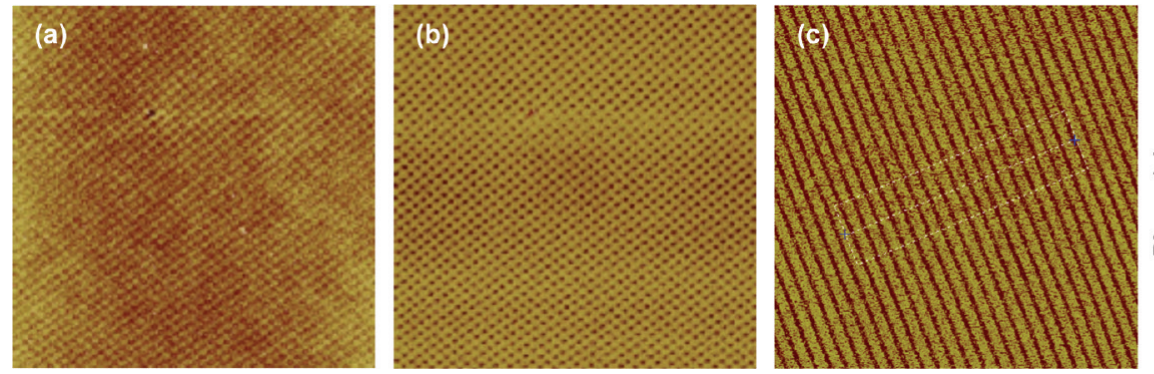

(d)

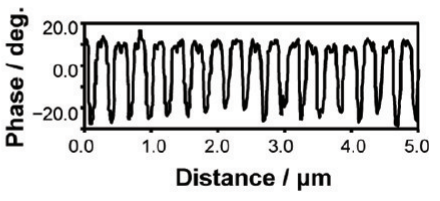

Figure 5. AFM Tapping Mode images of samples formed by carrying out interferometric exposures of OEG-terminated SAMs, followed by subsequent immersion of the samples in solutions of bovine serum albumin. (a) Topographical and (b) phase images of a sample patterned using two orthogonal exposures. (c) Phase image of lines formed in a single exposure and (d) cross section through a region in (c). Image sizes: (a) $10 \times 10 \mu \mathrm{m}^{2}, z$-contrast range $0-7 \mathrm{~nm}$ dark to bright; (b) $10 \times 10 \mu \mathrm{m}^{2}, z$-contrast range $0-120^{\circ}$ dark to bright; (c) $7.4 \times 7.4 \mu \mathrm{m}^{2}, z$-contrast range $0-120^{\circ}$ dark to bright.

carbon atoms in the alkyl chain linking the OEG terminal group to the surface and the ether carbon atoms in the OEG group itself. Following exposure to $2.2 \mathrm{~J} \mathrm{~cm}^{-2}$ of UV light (Figure $4 \mathrm{~b}$ ), new components were evident in the $\mathrm{C} 1 \mathrm{~s}$ spectrum that may be attributed to the presence of aldehydes $(287.8 \mathrm{eV})$ and carboxylates $(289.4 \mathrm{eV})$.

C 1s spectra were acquired for samples exposed to a range of exposures up to $91 \mathrm{~J} \mathrm{~cm}^{-2}$. The intensities of the ether, aldehyde, and carboxylate components in the fitted spectra are shown as a function of exposure in Figure 4c. As the UV exposure increased, the relative magnitude of the ether component decreased, falling rapidly to ca. $50 \%$ of its original magnitude after exposures of little more than $1 \mathrm{~J} \mathrm{~cm}^{-2}$. The aldehyde component was evident at significantly smaller exposures, however, and after an exposure of only $0.3 \mathrm{~J} \mathrm{~cm}^{-2}$, its area was ca. $11 \%$ that of the $\mathrm{C} 1 \mathrm{~s}$ peak. The increase in the area of the aldehyde peak was approximately equal to the decrease in the area of the ether component at these low doses, suggesting direct conversion of OEG units to aldehydes. However, as the exposure increased further, the area of the aldehyde peak varied comparatively little, while the area of the ether component decreased substantially. Instead, the area of the carboxylate peak increased as the exposure became larger. It is likely that the carboxylates result from photo-oxidation of the aldehydes, and the comparative invariance of the area of the aldehyde component results from the attainment of a steady state in which the creation of aldehydes by degradation of OEG units is balanced by their consumption through oxidative conversion to carboxylates. Previous work shows that the exposure required for photooxidation of the sulfur atoms in the OEG headgroup is ca. $10 \mathrm{~J}$ $\mathrm{cm}^{-2}{ }^{26}$ The data in Figure 4c show that there is extensive degradation, and extensive creation of aldehyde groups, at doses less than one-tenth that value, i.e., at exposures that leave the majority of the adsorbate sulfur-gold bonds intact.

To test their suitability for nanometer scale patterning, OEGterminated SAMs were exposed by IL, using the Fresnel mirror apparatus. After exposure, the samples were immersed in solutions of bovine serum albumin (BSA) in phosphate-buffered saline solution $\left(1 \mathrm{mg} \mathrm{mL}^{-1}\right)$ and then rinsed with buffer. The samples were imaged by AFM in tapping mode. Figure 5a,b shows samples that were patterned using two orthogonal exposures to create orthogonal lines of modified material enclosing square regions of unmodified SAM. In this case, the modified regions were no longer protein-resistant, so the images showed orthogonal lines of protein surrounding globular regions of bare, unmodified surface. The pitch was $280 \mathrm{~nm}$. Figure 5a is a height image, and Figure $5 \mathrm{~b}$ is a phase image. The protein features, which are raised above the level of the intact SAM and thus have bright contrast in the height images, also exhibit bright contrast in the 

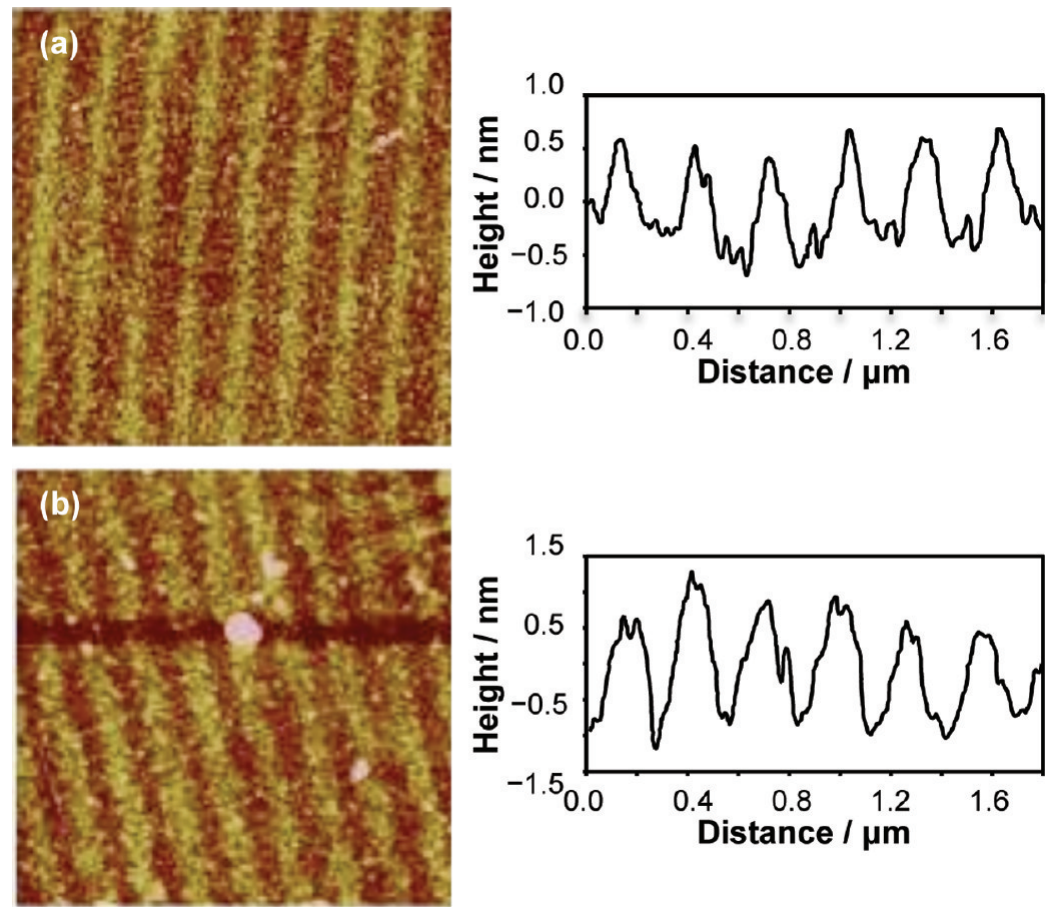

Figure 6. Tapping Mode AFM images and line sections of IL-patterned OEG-terminated SAMs following (a) immersion in a solution of streptavidin alone and (b) immersion in streptavidin followed by a solution of biotinylated IgG. Both images are $2.5 \times 2.5 \mu \mathrm{m}^{2}$.

phase image because the protein molecules are more viscous than the adsorbates in the bare SAM regions. Higher viscosity results in greater energy dissipation in the tip-sample contact, giving rise to a larger phase lag and hence brighter contrast in the phase image. ${ }^{55,56}$

Figure $5 \mathrm{c}$ shows a phase image of a sample that has been patterned in a single exposure, together with a line section (Figure $5 \mathrm{~d}$ ). The pitch was $282 \mathrm{~nm}$. The fwhm of the bare regions was $97 \mathrm{~nm}$, while that of the protein-covered bars was $185 \mathrm{~nm}$.

In IL, the sample is subjected to a continuous gradient of light intensity, and so it might be expected that low densities of aldehyde groups would be generated even in the regions exposed to a low intensity of illumination. Proteins adsorb tenaciously to surfaces exhibiting low defect densities, and moreover, the presence of aldehyde groups that can covalently bind proteins might be expected to cause protein attachment across much of the sample surface. A density of aldehyde groups as few as one surface site in 100 might be sufficient to ensure a high protein coverage at the surface. However, the fact that clear, well-defined protein structures are observed in Figure 5 indicates that the low densities of defects created near intensity minima in the interference pattern do not bind proteins. The most likely explanation for this is that at low densities the degraded (i.e., shorter) adsorbate chains are "screened" by surrounding intact adsorbates, preventing them from binding to proteins. This introduces a nonlinearity into the exposure process, such that a threshold level of degradation must be reached before protein attachment becomes significant.

A key requirement for any protein patterning method is that the biomolecules retain their biological activity after patterning. To test whether proteins attached to IL-patterned OEG-terminated SAMs could retain their biological function, a sample was immersed in a solution of streptavidin following exposure. The resulting structures were imaged by AFM. Figure 6a shows a height image of the sample. Parallel, raised lines, corresponding to immobilized

(55) Magonov, S. N.; Elings, V.; Whangbo, M.-H. Surf. Sci. 1997, 375, 385

(56) Nolan, B. H.; Roger, E. M. J. Biomed. Mater. Res. 2000, 51, 307. protein molecules, were seen. Analysis of line sections indicated that the pitch was $284 \mathrm{~nm}$ and the fwhm of the protein structures was $101 \mathrm{~nm}$, consistent with the immobilization of streptavidin. The sample was then immersed in a solution of biotinylated mouse antihuman IgG. Because protein-protein interactions are weak, IgG would not be expected to bind to streptavidin unless a suitable recognition-mediated binding process was available. Streptavidin has four binding sites for biotin, so binding of the biotinylated protein would indicate retention of structure by the streptavidin after immobilization at the surface. Figure $6 \mathrm{~b}$ shows a height image of the structures in Figure 6a following exposure to biotinylated IgG. Analysis of the lines section indicated that the amplitude (i.e., peak-to-trough distance) had approximately doubled, from 1 to $2 \mathrm{~nm}$, consistent with results reported in an earlier study of micrometer-scale patterning using UV degradation of (1-mercaptoundec-11-yl)tri(ethylene glycol) $\mathrm{SAMs}^{34}$ and strongly suggesting that biotinylated IgG had bound to the lines of streptavidin formed by IL.

These data indicate that the streptavidin molecules in the patterns retained their biological recognition characteristics. The fwhm of the protein structures was also increased, from 101 to $137 \mathrm{~nm}$ (i.e., by an amount equivalent to $2-3$ times the protein dimensions). This increase was unexpected. It is possible that the biotinylated proteins extend beyond the edge of the regions functionalized by streptavidin, joined by the biotinylated linkers, meaning that the lines of streptavidin are effectively "swollen" by attachment of the biotinylated IgG along their edges.

\section{Conclusions}

Interferometric methods provide a simple, fast, and effective means of fabricating uniform patterned structures in self-assembled monolayers of alkanethiols, with high resolution over macroscopically extended areas. Direct oxidation of the thiol headgroup facilitates solution-phase exchange with contrasting thiols and enables the fabrication of lines of alternating surface free energy on both gold and palladium surfaces. Interferometric exposure of 
oligo(ethylene glycol)-terminated monolayers causes photodegradation of the OEG tail group at exposures an order of magnitude smaller than those required for complete oxidation of the headgroup. The photodegradation introduces aldehyde groups that bind proteins via imine bond formation with free amine groups. Protein patterns formed by this method retain their biological function, as demonstrated by the binding of biotinylated proteins to streptavidin nanolines. The present work has used only a simple two-beam configuration, but many other possibilities exist suggesting that interferometric methods provide a straight- forward route to a very wide range of surface patterns. Interferometric methods offer great promise for bionanofabrication in laboratories that otherwise possess very little infrastructure for nanofabrication.

Acknowledgment. G.T., J.A., and G.J.L. thank EPSRC (Grants EP/D064767/1 and GR/C523857/1) for financial support. J.A. thanks the University of Sheffield for a travel grant. G.P.L. and S.R.J.B. thank the National Science Foundation (Grants 0515684 and 0611616) for financial support. 